\title{
A Study on Bi HX Group
}

\section{P.Pandiammal \\ Department of \\ Mathematics, PSNA CET, \\ Dindigul, Tamil Nadu, India}

\author{
R.Natarajan \\ HOD, Department of \\ Mathematics, Alagappa \\ University, \\ Karaikudi-630 003, Tamil \\ Nadu, India
}

\author{
N.Palaniappan \\ Department of \\ Mathematics, Alagappa \\ University, \\ Karaikudi-630 003, Tamil \\ Nadu, India
}

\begin{abstract}
In this paper we introduce the concept of Bi HX group and study some of its properties.

Keywords

Bi group, Sub-bi group, HX group, Bi HX group, HX group homomorphism, Bi HX group homomorphism.
\end{abstract}

\section{INTRODUCTION}

The theoretical needs of the set-value mappings lead the birth of some mathematical structures. Prof. Li Hongxing [1,2] first introduced the concept of HX group which originated the study of HX group; moreover, some useful results are obtained. Since the operations in a HX group is based on the operations of some elements in the base algebra, it is worth to study how to represent directly these operations and to judge whether a subset of the power set are a certain algebraic structure. In this paper we introduce and discuss the properties of Bi HX group.

\section{PRELIMINARIES}

\section{Definition 1.1}

A set $(\mathrm{G},+, \mathrm{o})$ with two binary operation ' + ' and 'o' is called a $\mathrm{Bi}$ group if there exists two proper subsets $G_{1 \text { and }} G_{2}$ of G such that,

$$
\begin{aligned}
& \text { (i) }\left(G_{1},+\right) \text { is a group, } \\
& \text { (ii) }\left(G_{2}, \text { o }\right) \text { is a group, } \\
& \text { (iii) } G=G_{1} \cup G_{2}
\end{aligned}
$$

Definition 1.2. A non-empty subset $\mathrm{H}$ of a bi group $(\mathrm{G},+, \mathrm{o})$ is called a sub- bi group, if $\mathrm{H}$ itself is a bi group under a operation '+' and 'o' defined on G.

Definition 1.3. [1] In $2^{\mathrm{G}}-\{\phi\}$ we define an algebraic operation: $A B=\{a b / a \in A, b \in B\}$
An nonempty set $g \subset 2^{\mathrm{G}}-\{\phi\}$ is called a HX group on $\mathrm{G}$, if $g$ is a group with respect to the operation (I), which its unit element is denoted by E.

Definition 1.4. Let $g$ be a HX group on $\mathrm{G}$. Let us defined that,

$$
\begin{aligned}
& \mathrm{G}^{*}=\bigcup\{\mathrm{A} / \mathrm{A} \in \mathrm{g}\} \\
& G^{o}=\left\{a \in G^{*} / a^{-1} \in G^{*}\right\}
\end{aligned}
$$

Definition 1.5. A set $(g,+, o)$ with two binary operation "+" and "o" is called a Bi HX group if there exists two proper subsets $g_{1} \& g_{2}$ such that

(i) $\quad\left(g_{1,+}\right)$ is a HX group

(ii) $\quad\left(g_{2}, 0\right)$ is a HX group

(iii) $\quad g=g_{1} \cup g_{2}$

Definition 1.6. Let $g$ and $g^{1}$ be any two HX groups. A mapping $\mathrm{f:} g \rightarrow \mathrm{g}^{1}$ is called a HX group homomorphism if it satisfies the condition,

$$
f(A B)=f(A) f(B) \text { for all } A, B \in g \text {. }
$$

Definition 1.7. Let $\left(g=g_{1} \cup g_{2},+, 0\right)$ and $\left(g^{1}=\right.$ $\left.g_{1}{ }^{1} \cup g_{2}{ }^{1},+', o^{\prime}\right)$ be a Bi HX group. The map f: $\mathrm{g} \rightarrow \mathrm{g}^{1}$ is said to be an Bi HX group homomorphism if $\mathbf{f}$ is restricted to $g_{1}$ (i.e) $\mathbf{f} / g_{1}$ is a HX group homomorphism from $g_{1}$ to $g_{1}{ }^{1}$ and $f$ is restricted to $g_{2}$ (i.e) $\mathbf{f} / g_{2}$ is a $\mathrm{HX}$ group homomorphism from $g_{2}$ to $9_{2}{ }^{1}$. 
Definition 1.8. Let $\left(g=g_{1} \cup g_{2},+, 0\right)$ be a Bi HX group of a bi group $\mathrm{G}$.

$$
\begin{aligned}
& \text { Then define } \mathrm{G}^{*}=\mathrm{G}_{1}{ }^{*} \bigcup \mathrm{G}_{2}{ }^{*} \\
& \text { Where } \mathrm{G}_{1}{ }^{*}=\bigcup\left\{\mathrm{A} / \mathrm{A} \in g_{1}\right\} \text { and } \\
& \mathrm{G}_{2}{ }^{*}=\bigcup\left\{\mathrm{A} / \mathrm{A} \in g_{2}\right\} \\
& G^{o}=G_{1}^{o} \bigcup G_{2}^{o} \text { where } \\
& G_{1}^{o}=\left\{a \in G_{1}^{*} / a^{-1} \in G_{1}^{*}\right\} \text { and } \\
& G_{2}^{o}=\left\{a \in G_{2}^{*} / a^{-1} \in G_{2}^{*}\right\}
\end{aligned}
$$

\section{BASICS THEOREM ON HX GROUP:}

Theorem2.1. [1] If $g$ is a HX group on $G$, then

$$
\text { (i) } \quad(\forall A \in g)(|A|=|B|) \text {; }
$$

(ii) $\forall A, B \in g)$

$$
(A \cap B \neq \phi \Rightarrow|A \cap B|=|E|)
$$

Proof. (i) In one respect we have

$\mathrm{AE}=\mathrm{A}$

$\Rightarrow(\forall a \in A)(a E \subset A E=A)$

$\Rightarrow|E|=|a E| \leq|A|$

In the other respect we have

$$
\begin{aligned}
& \mathrm{A} \mathrm{A}^{-1}=\mathrm{E} \\
& \Rightarrow\left(\forall b \in \mathrm{A}^{-1}\right)\left(b A \subset \mathrm{A}^{-1} A=E\right) \\
& \Rightarrow|A|=|b A| \leq|E|
\end{aligned}
$$

(ii) First $|A \cap B| \leq|A|=|E|$

Second, $c \in A \cap B \Rightarrow c E \subset A \cap B \Rightarrow$ $|E|=|c E| \leq|A \cap B|$

Theorem2.2. [1] Let $H$ be a subgroup of $G$ and $E$ be a subset of $G$ satisfying $E^{2}=E$. If $(\forall a \in H)(a E=E a)$ then $g=\{\mathrm{aE} / \mathrm{a} \in H\}$ is a HX group on $\mathrm{G}$ its unit element just $\mathrm{E}$.
Proof. Take the surjection $\mathrm{f}: \mathrm{H} \rightarrow \mathrm{g}, a \mapsto a E$.

$f(a b)=(a b) E$

$$
\begin{aligned}
& =(a b) E E \\
& =a(b E) E \\
& =a(E b) E \\
& =(a E)(B e) \\
& =f(a) f(b),
\end{aligned}
$$

So, $\mathrm{H} \sim \mathrm{g}$. This $\mathrm{g}$ is a group. Moreover, $f(e)=e E=E$. So $E$ is the unit element of $g$.

Theorem2.3. [1] Let $g$ is a HX group on G. If $E$ is a subgroup of $\mathrm{G}$, then(i) $\mathrm{g}=\{\mathrm{aE} / \mathrm{a} \in \mathrm{G} *\}$;

(ii) $\quad G^{*}$ if a subgroup of $G$.

Proof. (i) $\forall A \in$ g, take $a \in \mathrm{A}$. We have $\mathrm{aE} \subset A E=A$. It can be proved that $\mathrm{aE}=\mathrm{A}$. If it is not true, then there exists $\mathrm{b} \in A-a E$. Then we have $a^{-1} b \notin E$ because $\quad b=a c \in a E \quad$ if $a^{-1} b=c \in E$. If $d \in A^{-1}$ we have da and $\mathrm{db} \in A^{-1} A=E$.

Thus $a^{-1} b=a^{-1} d^{-1} d b$

$$
=(d a)^{-1}(d b) \in E \text {. }
$$

This is a contradiction with $a^{-1} b \notin E$. So $\mathrm{aE}=A$. This means that $\mathrm{g} \subset\{\mathrm{aE} / \mathrm{a} \in \mathrm{G} *\}$.

Conversely, $\forall a \in G^{*}, \exists A \in \mathrm{g}$, such that $\mathrm{a} \in \mathrm{A}$. So $\mathrm{aE}=A \in$ g. Thus $\{\mathrm{aE}$ $/ \mathrm{a} \in \mathrm{G} *\} \subset \mathrm{g}$.

(ii) $\forall a \in G^{*}, \exists A \in \mathrm{g}$, such that $\mathrm{a} \in \mathrm{A}$. Noting $e \in E$ and $A A^{-1}=E$, then there exist $\mathrm{b} \in A, b^{-1} \in A^{-1}$, such that $b b^{-1}=e$. From $\mathrm{A}=\mathrm{bE}$ we have $c \in E$ such that $\mathrm{a}=\mathrm{bc}$. So, $a^{-1}=(b c)^{-1}$ $=c^{-1} b^{-1} \in E A^{-1}=A^{-1} \subset G^{*}$ 
Theorem2.4. [1] Let $\mathrm{f}$ be a homomorphism from $\mathrm{G}$ to another group $G^{\prime}$. We have

(i) If $g$ is a HX group on $\mathrm{G}$, then $g^{1}=\{\mathrm{f}(\mathrm{A}) / \mathrm{A} \in \mathrm{g}\}$ is a HX group on $G^{\prime}$ and $g \sim \mathrm{g}^{1}$

(ii) Let $\mathrm{f}$ be a surjection. If $\mathrm{g}^{1}$ is a HX group on $G^{\prime}$, then

$\mathrm{g}=\left\{\mathrm{f}^{-1}\left(A^{\prime}\right) / A^{\prime} \in \mathrm{g}^{1}\right\}$ is a HX group on $G$ and $g \sim g^{1}$

Proposition 2.1. [1] Let $g$ be a HX group on $G$, and $\mathrm{B} \subset 2^{\mathrm{G}}-\{\phi\}$ with $\mathrm{B}^{2}=\mathrm{B}$. If $\mathrm{B}$ satisfies the condition: $(\forall A \in g)(A B=B A)$, then

$$
g_{B}=\{A B / A \in g\} \text { is a HX group }
$$

on $\mathrm{G}$ and $\mathrm{g}-\mathrm{g}_{\mathrm{B}}$.

The proof is straight.

\section{SOME RESULTS ON Bi-HX GROUP:}

Theorem 3.1. If $\mathrm{g}$ is a $\mathrm{Bi} \mathrm{HX}$ group on a $\mathrm{Bi}$ group $\mathrm{G}$, then

$$
\begin{gathered}
\text { (i) }(\forall A \in g)(|A|=|B|) \\
\text { (ii) }(\forall A, B \in g) \\
(A \cap B \neq \phi \Rightarrow|A \cap B|=|E|)
\end{gathered}
$$

Proof. (i) $A \in g=g_{1} \cup g_{2}$.

Let $A \in g_{1}$ and $g_{1}$ is a HX group on $G_{1}$.

Therefore, $|A|=|B|$

Similarly, $A \in g_{2}$ and $g_{2}$ is a $H X$ group on $G_{2}$.

$$
|A|=|B|
$$

Hence $(\forall A \in g)(|A|=|B|)$

Let $A, B \in g_{1}$. Since $g_{1}$ is a $H X$ group on $G_{1}$

$$
|A \cap B|=|E|
$$

Similarly, $A, B \in g_{2}$. Since $g_{2}$ is a $H X$ group on $G_{2}$

$$
\text { Therefore }|A \cap B|=|E|
$$

Hence

$(\forall A, B \in g)$

$$
(A \cap B \neq \phi \Rightarrow|A \cap B|=|E|) .
$$

Theorem 3.2. Let $g=\left(g_{1} \cup g_{2},+, 0\right)$ be a $\mathrm{Bi}$ HX group of a bi group $G=\left(G_{1} \cup G_{2},+, o\right)$. Then

(a) $\mathrm{G}^{*}$ is a sub-bi group of $\mathrm{G}$

(b) $G^{o} \neq \phi \quad$ iff $\quad e_{1} \in G^{o}$ and $e_{2} \in G^{o}$ where $e_{1}$ is the identity element of $\mathrm{G}_{1}$ and $e_{2}$ is the identity element of $\mathrm{G}_{2}$ respectively.

(c) $G^{o} \neq \phi$ iff $G^{o}$ is a sub-bi group of G.

Proof. (a) Let $a, b \in G^{*}$. Then

(i) $\mathrm{a}, \mathrm{b} \in \mathrm{G}_{1}{ }^{*}$, then there exists $A, B \in \mathrm{g}_{1}$ such that $\mathrm{a} \in \mathrm{A}$ and $\mathrm{b} \in \mathrm{B}$.

$$
\begin{aligned}
& \Rightarrow \mathrm{a}+\mathrm{b} \in \mathrm{A}+\mathrm{B} \subset \mathrm{G}_{1}{ }^{*} \\
& \Rightarrow\left(\mathrm{G}_{1}{ }^{*},+\right) \text { is a group. }
\end{aligned}
$$

(ii) c, $\mathrm{d} \in \mathrm{G}_{2}{ }^{*}$, then there exists $C, D \in \mathrm{g}_{2}$ such that $\mathrm{c} \in \mathrm{C}$ and $\mathrm{d} \in \mathrm{D}$.

$$
\begin{aligned}
& \Rightarrow \operatorname{cod} \in \mathrm{CoD} \subset \mathrm{G}_{2}{ }^{*} \\
& \Rightarrow\left(\mathrm{G}_{2}{ }^{*}, \mathrm{o}\right) \text { is a group. }
\end{aligned}
$$

Clearly $\mathrm{G}^{*}=\mathrm{G}_{1}{ }^{*} \cup \mathrm{G}_{2}{ }^{*}$

Therefore, $G^{*}$ is a sub-bi group of $G$.

(b) Obvious

(c) Let $G^{o} \neq \phi$

Let $\mathrm{a}, \mathrm{b} \in G^{o}$

(i)

$$
\begin{aligned}
& \text { Let } \mathrm{a}, \mathrm{b} \in G_{1}^{o} \\
\Rightarrow & -\mathrm{a}, \mathrm{b} \in \mathrm{G}_{1}{ }^{*} \\
\Rightarrow & -(\mathrm{a}+\mathrm{b}) \in \mathrm{G}_{1}{ }^{*} \\
\Rightarrow & \mathrm{a}+\mathrm{b} \in G_{1}^{o}
\end{aligned}
$$

Therefore $\left(G_{1}^{o},+\right)$ is a group. 
(ii) Let $\mathrm{a}, \mathrm{b} \in G_{2}^{o}$

$$
\begin{aligned}
& \Rightarrow \mathrm{a}^{-1}, \mathrm{~b}^{-1} \in \mathrm{G}_{2}{ }^{*} \\
& \Rightarrow(\mathrm{ab})^{-1} \in \mathrm{G}_{2}{ }^{*} \\
& \Rightarrow \mathrm{ab} \in G_{2}^{o} \\
& \Rightarrow\left(G_{2}^{o},+\right) \text { is a group. }
\end{aligned}
$$

Therefore $G^{o}=G_{1}^{o} \bigcup G_{2}^{o}$ is a sub-bi group of $\mathrm{G}$.

Theorem3.3. Let $\mathrm{f}$ be a bi group homomorphism from $\mathrm{G}$ to another bigroup $G^{\prime}$. We have

(i) If $g$ is a Bi HX group on a bi group $\mathrm{G}$, then $g^{1}=$ $\{\mathrm{f}(\mathrm{A}) / \mathrm{A} \in \mathrm{g}\} \quad$ is a $\mathrm{Bi} \mathrm{HX}$ group on $G^{\prime}$ and $g \sim \mathrm{g}$ 1

(ii) Let $\mathrm{f}$ be a surjection. If $\mathrm{g}^{1}$ is a $\mathrm{Bi} \mathrm{HX}$ group on $G^{\prime}$, theng $=\left\{\mathrm{f}^{-1}\left(A^{\prime}\right) / A^{\prime} \in g^{1}\right\}$ is a $\mathrm{Bi} \mathrm{HX}$ group on $G$ and $g \sim g^{1}$.

\section{CONCLUSIONS}

Further work is in progress in order to develop the Fuzzy Bi-HX group and Anti Fuzzy Bi-HX group.

\section{REFERENCES}

[1] LiHongxing, HX group, BESEFAL, pp(31-37).

[2] LiHongxing, Hypergroup (I), BESEFAL, 23(1985), pp(22-29).

[3] Hall,M., The Theory of Group, Macmillan, New York, 1959. 